\title{
Reliability and Congestion Control in Cognitive Radio Sensor Networks
}

\author{
A. Ozan Bicen*, Ozgur B. Akan \\ Next-generation Wireless Communications Laboratory, \\ Department of Electrical and Electronics Engineering, Koc University, Istanbul, 34450, Turkey.
}

\begin{abstract}
Communication requirements for cognitive radio sensor networks (CRSN) necessitate addressing the problems posed by dynamic spectrum access (DSA) in an inherently resource-constrained sensor networks regime. In this paper, arising challenges for reliability and congestion control due to incorporation of cognitive radio capability into sensor network are investigated along with the open research issues. Impact of DSA, i.e., activity of licensed users, intermittent spectrum sensing and spectrum handoff functionalities based on spectrum availability, on the performance of the existing transport protocols are inspected. The objective of this paper is to point out the urgent need for a novel reliability and congestion control mechanism for CRSN. To this end, CRSN challenges for transport layer are revealed and simulation experiments are performed to demonstrate the performance of the existing transport protocols in CRSN.
\end{abstract}

Keywords: Cognitive radio, transport layer, reliability, congestion control, rate control, sensor networks

\section{Introduction}

Spectrum utilization inefficiency with increasing usage of wireless communications has triggered the development of dynamic spectrum access schemes. The key enabling technology providing dynamic, i.e., opportunistic, spectrum access is the cognitive radio (CR) [1-5]. Cognitive radio has the capability to sense the spectrum and determine the vacant bands. Cognitive radio can make use of these available bands in an opportunistic manner surpassing the traditional fixed spectrum assignment approach in terms of overall spectrum utilization.

In licensed bands, wireless users with a specific license to communicate over the allocated band, i.e., the licensed user, has the priority to access the channel. Upon these natural habitants of a specific frequency band start communication; the cognitive radio users must detect the potentially vacant bands, through spectrum sensing. Then, they decide on which channels to move, i.e., perform spectrum decision. Finally, they adapt their transceiver so that the active communications are continued over the new channel as a result of spectrum handoff. This operation sequence is called cognitive cycle [2, 4, 6], and it can also be applied for an access to an unlicensed band by cognitive radios, i.e., unlicensed users, which have the same priority to use the unlicensed channel.

The capabilities of cognitive radio may provide many of the current wireless systems with adaptability to existing spectrum allocation in the deployment field, and hence,

\footnotetext{
*Corresponding Author. Tel. +90 2123382645.

Email addresses: abicen@ku.edu.tr (A. Ozan Bicen), akan@ku.edu.tr (Ozgur B. Akan)
}

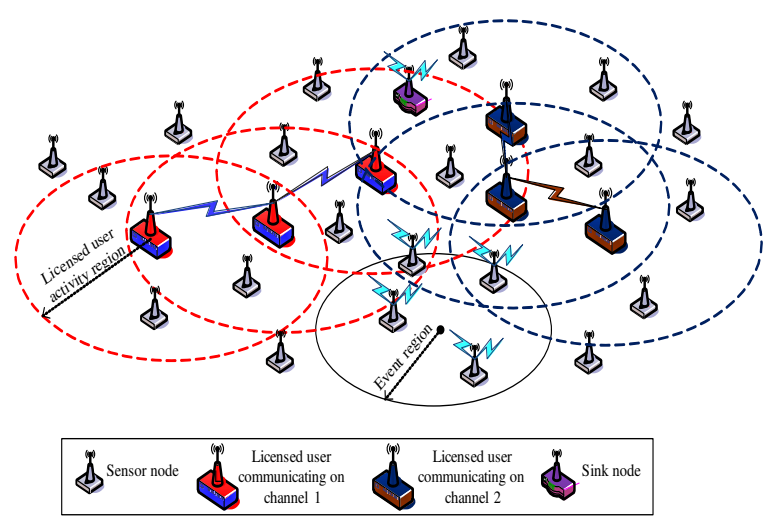

Figure 1: CRSN topology.

improve overall spectrum utilization. Furthermore, these capabilities may provide efficient utilization of limited resources of sensor nodes in terms of communication and processing in wireless sensor networks (WSN). In fact, a WSN comprised of sensor nodes equipped with cognitive radio may benefit from the potential advantages of the salient features of dynamic spectrum access (DSA), and therefore, this defines a new sensor network paradigm, i.e., cognitive radio sensor networks (CRSN) [7]. CRSN provides dynamic spectrum access in contrast to common fixed spectrum utilization of WSN.

CRSN nodes forming a network topology is shown in Fig. 1. The main duty of the sensor nodes is to perform sensing on the environment. In CRSN, depending on the spectrum availability, sensed data is transmitted to the sink in a multi-hop manner. Other than the event read- 
ings, sensor nodes may also require to exchange control data for licensed user activity detection, spectrum allocation, spectrum sensing, and spectrum coordination.

While the potential advantages of CRSN stand as a significant enhancement over traditional sensor networks, the realization of CRSN depends on addressing many difficult challenges, posed by the unique characteristics of both cognitive radio and sensor networks, and further amplified by their union. One of them is reliable event detection and tracking with congestion control, which is, in general, responsibility of transport layer. In order to conserve limited network and energy resources of sensor nodes and provide reliable event delivery, sufficient amount of information must be delivered to the sink while preventing congestion. However, the existing work on the reliable delivery of event features and congestion control in sensor networks do not consider challenges posed by the dynamic spectrum access. Furthermore, there is no specific solution for CRSN to this date. Hence, as the first step for the design of new transport layer solutions, it is imperative to understand the challenges and observe the shortcomings of these existing solutions through a comprehensive set of simulation experiments. Recently, there has been some efforts to reveal challenges of cognitive radio networks for transmission control protocol (TCP) [8-11]. To the best of our knowledge, there exists no such study for sensor networks in the current literature.

In this paper, we introduce the main design challenges and principles for reliability and congestion control in CRSN. The existing communication protocols and algorithms devised for cognitive radio ad hoc networks as well as WSN are explored from the perspective of CRSN. Our objective is to acquaint CRSN challenges for transport layer, evaluate and reveal the performance and shortcomings of the existing transport protocols in CRSN communication scenarios. Additionally, the open research avenues for the realization of energy-efficient and reliable data communication in CRSN are highlighted.

The remainder of the paper is organized as follows. In Section II, the transport layer issues in CRSN, challenges and overview of existing protocols are given. In Section III, results of simulation experiments and the effects of cognitive cycle on reliable data delivery with congestion control in CRSN are presented. Performance evaluation results are discussed, and the open research challenges for design of CRSN transport protocols are explored in Section IV. Finally, we state the concluding remarks in Section V.

\section{Transport Layer in Cognitive Radio Sensor Net- works}

Transport protocols in wireless sensor networks (WSN) are primarily responsible for reliable and energy-efficient delivery of sensed event from source to sink. As well as considering quality of service (QoS) requirements of applications, congestion avoidance must be provided to preserve limited resources of sensor nodes in terms of pro- cessing, memory, communication, and more importantly, energy. CRSN is proposed to overcome spectrum scarcity and congestion problem caused by dense deployment of sensor nodes with opportunistic communication over licensed (and unlicensed) bands in WSN [7]. In addition to application-based reliability and energy-efficient communication notion in WSN, opportunistic spectrum access challenge emerges in CRSN. Intrinsically, transport protocols designed for traditional WSN do not take DSA into consideration. However, additional link delays and packet losses as a result of varying spectrum availability, spectrum mobility and spectrum sensing are the key issues for reliability and congestion control in CRSN.

In this section, we investigate the specific CRSN challenges for reliable and energy-efficient data delivery and explore the existing transport solutions developed for WSN and cognitive radio ad hoc networks together.

\subsection{CRSN Challenges}

To provide successful event detection and tracking in CRSN, sensed phenomena by sensor nodes must be delivered to the sink in accordance with the application-specific requirements such as number of delivered packets, delay bound, jitter, end-to-end delivery, etc. Additionally, this must be accomplished without interfering with licensed users and providing congestion avoidance for effective usage of limited resources of sensor nodes.

Clearly, in addition to inherent WSN demands, CRSN imposes unique challenges due to DSA functionalities, which are outlined as follows.

\subsubsection{Intermittent Communication due to Spectrum Sens- ing}

Silent periods of spectrum sensing prevents sensor nodes to deliver detected event to the sink. During spectrum sensing periods, sensor nodes do not transmit their collected information, since existing radio frequency modules cannot provide sensing and transmission functionalities at the same time. Thus, event transport is periodically disrupted in CRSN. Moreover, spectrum sensing duration brings a trade-off between false alarm and throughput. While a shorter sensing duration may result in an increase in the number of transmitted packets, inaccurate detection of licensed user activity may severely affect the transport layer.

\subsubsection{Connection Blackout due to Spectrum Handoff}

Upon licensed user activity detection, unlicensed users are required to vacate the channel. Until a vacant channel is found and spectrum handoff is performed by all sensor nodes participating in the event delivery, sink connection is disrupted due to spectrum unavailability. Connection blackout lasts depending on the licensed user activity, and event information cannot be delivered to the sink during blackouts. 


\subsubsection{Spiky End-to-end RTT due to Dynamic Spectrum Availability}

Together with the extra delay introduced by cognitive cycle functionalities, limited spectrum availability and increasing licensed user activity may amplify round trip time (RTT). Due to dynamically varying nature of licensed user activity, there may be large variations on the spectrum availability. Sudden decrease in spectrum availability, i.e., sharp increase in licensed user arrival rate or duration, is an expected situation in CRSN. Therefore, abrupt variation on the link level and end-to-end RTT estimates may be observed in CRSN. Additionally, based on varying interference limitations with licensed users at different channels, spectrum sensing duration may vary [12, 13] and further complicate estimation of RTT. Moreover, spectrum handoff mechanism also imposes additional delay on communication due to change of operating frequency. These various effects complicate the estimation of RTT. Thus, incorrect estimation of RTT may prevent the delay-based congestion control and rate adjustment mechanisms operate correctly, and hamper the performance of transport layer. In addition, achieving real-time communication in CRSN is quite challenging due to spiky end-to-end RTT imposed by DSA.

\subsubsection{Packet Losses due to Spectrum Mobility}

Spectrum mobility may cause packet drops due to link layer buffer overflows based on spectrum availability and connectivity. Due to intermittent link availability, link layer buffer fills up with the packets generated and coming from other nodes. Link layer queue overflows with the generation of new packets containing event readings and causes loss of event information, which is undesirable in WSN. On the other hand, spectrum mobility evokes congestion due to varying channel conditions and the need for coordination while connection establishment at the new operating channel. Due to bursty nature of sensor network, this connection establishment phase may result in excessive packet losses.

\subsubsection{Varying Network Capacity}

Event delivery performance is dependent on the capacity of network, which is varying due to DSA in CRSN. Depending on the network capacity, number of event reporting nodes and their reporting rates may need to be adjusted to avoid congestion. Moreover, network capacity may limit reliability in event detection and tracking, i.e., achievable maximum number of delivered packets and minimum delay bound may not satisfy QoS requirements of applications.

\subsubsection{Need for Synchronization of Transmission Periods}

Due to spatially varying licensed user activity, different sensing durations may be required at different regions of the network. While event information is being delivered to the sink, event-to-sink path may traverse along the regions with different spectrum sensing durations. Therefore, to deliver packets to the next node, sending node should be aware of sensing periods of the next node. Otherwise, while spectrum sensing, next node will not receive sent packets.

\subsection{Overview of Existing Transport Protocols}

Considerable amount of research efforts have yielded many transport protocols addressing challenges posed by WSN paradigm. Main properties of the existing protocols are classified in Table 1 . In spite of providing congestion detection mechanisms and reliable transport functionalities, none considers both challenges of DSA and requirements of WSN.

Congestion Detection and Avoidance (CODA) protocol [14] presents an energy-efficient congestion control algorithm in order to prevent possible congestion on sourceto-sink path by regulating source sending rate. Eventto-Sink Reliable Transport (ESRT) [15] and Real-Time and Reliable Transport $\left((\mathrm{RT})^{2}\right)$ [16] protocols are ratebased mechanisms which provide congestion avoidance and event-to-sink reliability. Additionally, $(\mathrm{RT})^{2}$ has real-time support. On the other hand, Pump Slowly Fetch Quickly (PSFQ) [17] provides reliability on downstream path and uses hop-by-hop recovery to retrieve missing packets. Reliable Multi-Segment Transport (RMST) protocol [18] provides negative acknowledgement (NACK) based guaranteed delivery like PSFQ, and both of them point out the importance of hop-by-hop recovery ${ }^{1}$.

Recently, a window-based transport layer protocol addressing challenges of DSA in cognitive radio ad hoc networks, Transport Protocol for Cognitive Radio Ad-Hoc Networks (TP-CRAHN), is proposed in [19]. A crosslayer solution jointly considering the DSA functionalities, physical-layer modulation and coding scheme, and datalink layer frame size is presented in [20] to maximize TCP throughput. A set of protocols are derived from TCP in [21] via tunning for DSA compatibility to serve delaytolerant applications. Although those recently proposed algorithms incorporate cognitive radio functionalities, they are designed based on TCP congestion control mechanism, which has clearly been shown to have very poor performance in sensor networks [22].

Additionally, there has recently been active research on spectrum utilization efficiency and optimal spectrum sharing policies in cognitive radio networks [23-26]. In [23], a new approach for spectrum sensing is developed with an analytical model to estimate the power in a given channel and location due to nearby communication, and then, these are used to formulate optimal channel assignment problem within the mesh network. In [24], per-node based power control for multi-hop cognitive radio networks

\footnotetext{
${ }^{1}$ RMST [18] is not included in the performance evaluation as it aims to provide $100 \%$ reliability on a per packet basis, which is shown to be unsuitable for WSN [15].
} 
Table 1: Summary of Existing Transport Protocols

\begin{tabular}{|l|l|l|l|l|l|l|}
\hline Protocol & $\begin{array}{l}\text { Congestion } \\
\text { Detection }\end{array}$ & Flow & $\begin{array}{l}\text { Reliability } \\
\text { Support }\end{array}$ & $\begin{array}{l}\text { Real-time } \\
\text { Support }\end{array}$ & $\begin{array}{l}\text { DSA } \\
\text { Support }\end{array}$ & Scope \\
\hline$C O D A[14]$ & Active & Upstream & - & - & - & WSN \\
$E S R T[15]$ & Passive & Upstream & Event & - & - & WSN \\
$P S F Q[17]$ & - & Downstream & Packet & - & - & WSN \\
$(R T)^{2}[16]$ & Passive & Upstream & Event & + & - & WSN \\
$R M S T[18]$ & - & Upstream & Packet & - & - & WSN \\
$T P-C R A H N[19]$ & Active & Both & Packet & - & + & CRAHN \\
\hline
\end{tabular}

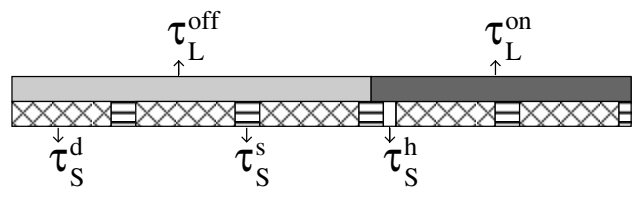

Figure 2: Licensed user and sensor node activity patterns with time.

is proposed, and a mathematical framework is developed to jointly optimize power control, scheduling, and routing. In order to increase data rates of secondary user communication sessions in a multi-hop cognitive radio network, joint optimization problem of power control, scheduling, and routing is studied in [25]. For video multicast in an infrastructure-based cognitive radio network coexisting with different licensed user networks, a cross-layer optimization approach is developed in [26] to optimize the overall received video quality, and provide proportional fairness among multicast users, while keeping the interference to licensed users below predefined constraints. Since these approaches are developed to increase end-to-end performance and spectrum efficiency in multi-hop cognitive radio networks, they do not consider neither many-to-one communication nature nor correlated data gathering of sensor networks.

Clearly, the existing transport protocols and spectrum sharing schemes are not specifically designed to achieve reliability in CRSN. To assess the performance of the existing transport protocols in CRSN, next, a wide range of simulations are performed and results are provided.

\section{Performance Evaluation}

Simulations are performed by extending NS-2 [27] with multichannel extensions to enable dynamic spectrum access and cognitive radio in sensor networks. 200 nodes and a sink are placed randomly in a $200 \mathrm{~m} \times 200 \mathrm{~m}$ field. 25 source nodes are randomly selected within an event area of radius $20 \mathrm{~m}$. Packet size is 30 bytes and nodes are equipped with a single transmitter/receiver, which has a radio range of $40 \mathrm{~m}$. DSR [28] is used as routing protocol and each node has a simple CSMA/CA based medium access control layer with a queue size of 65 .

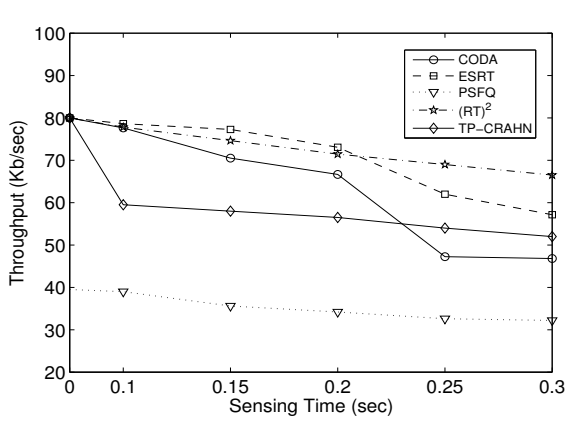

(a)

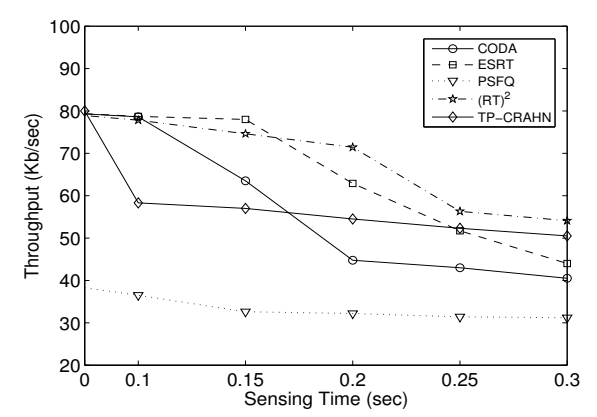

(b)

Figure 3: Variation of throughput while $\tau_{S}^{s}$ is varying from 0 to 0.3 sec for $\tau_{S}^{d}=3$ and $2 \mathrm{sec}$ and $\tau_{L}^{o n}=0$, in (a) and (b), respectively.

Licensed user activity is modeled with active $\left(\tau_{L}^{o n}\right)$ and inactive $\left(\tau_{L}^{o f f}\right)$ periods, and sensor node behavior is modelled with data transmission $\left(\tau_{S}^{d}\right)$, spectrum sensing $\left(\tau_{S}^{s}\right)$ and spectrum handoff $\left(\tau_{S}^{h}\right)$ periods as shown in Fig. 2. CODA [14], ESRT [15], $\mathrm{PSFQ}^{2}$ [17], $(\mathrm{RT})^{2}$ [16] and TPCRAHN [19] are included in the simulation experiments. Lastly, each simulation configuration is run 10 times and results are averaged.

\subsection{Spectrum Sensing in $C R S N$}

Effect of spectrum sensing period, i.e., transient lack of ability to transmit packets, on throughput is observed for varying $\tau_{S}^{s}$ from 0 to $0.3 \mathrm{sec}$ in the absence of licensed

\footnotetext{
${ }^{2} \mathrm{PSFQ}$ is tested on reverse direction from sink to source, different than other protocols.
} 


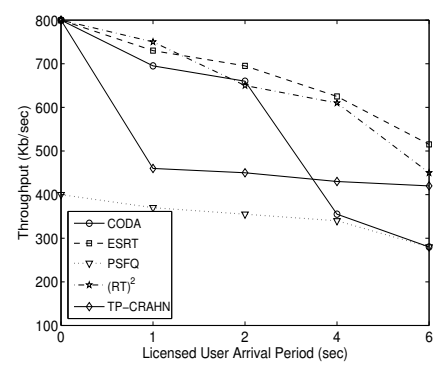

(a)

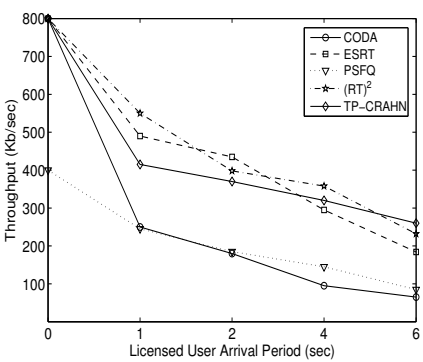

(b)

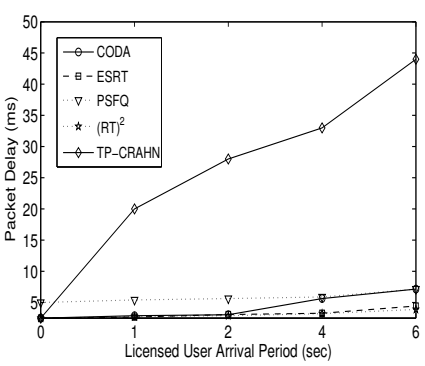

(c)

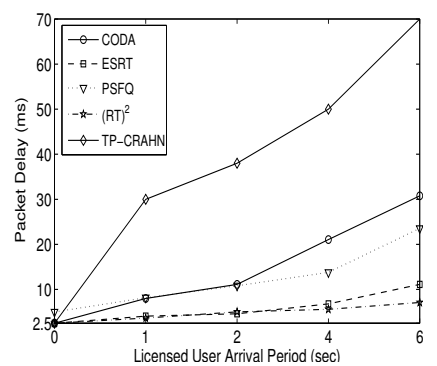

(d)

Figure 4: Variation of throughput, in (a) and (b), and delay, in (c) and (d), with varying $\tau_{L}^{\text {on }}$ from 0 to 6 sec, for constant $\tau_{L}^{o f f}=6$ and 3 sec, respectively.

user arrival. Fig. 3(a) and 3(b) show the throughput for $\tau_{S}^{d}=3$ and 2 sec, respectively.

Generally, all of the transport protocols exhibit a degradation in their throughput performance due to not considering silent sensing period. As spectrum sensing period increases, CODA, which uses feedback messages from intermediate nodes, sustains extra delays introduced by DSA. These delays cause highly variable RTT, and therefore, selective acknowledgement (SACK) packets are not received timely. This is interpreted as congestion and CODA decreases its rate to one half. It can be seen in Fig. 3(b) that throughput decreases about $40 \%$ with the decreasing data transmission period. ESRT and (RT) ${ }^{2}$ increase their reporting rate to obtain required reliability. They adjust transmission rate to compensate delay sustained in previous interval. However, due to misinterpretation of deficiency in reliability, inappropriate increase of transmission rate leads to congestion. On the other hand, PSFQ does not regulate its transmission rate, and hence, throughput performance degrades because of extra delay introduced by spectrum sensing, and there exists no mechanism considering this delay.

On the other hand, although initial drop, TP-CRAHN relatively maintains its performance as the sensing period increases. Especially, for higher spectrum sensing periods with lower data transmission period TP-CRAHN performs close to current WSN transport layer protocols. Since current WSN transport protocols are not aware of cognitive cycle, they attribute delays, retransmission timeouts and packet losses caused by DSA functionalities to bad channel condition or congestion, and perform inappropriate rate control operations according to their algorithm, which result in up to $50 \%$ performance loss.

In Fig. 3(a) and Fig. 3(b), it is observed that decreasing data transmission period and increasing spectrum sensing period have adverse effect on throughput, however, this effect should be minimized to satisfy reliability requirements while retaining energy-efficient operation.

Spectrum sensing, which is a part of cognitive cycle, has a great importance to detect licensed user activities. Sensor nodes, which are secondary users, also need to sense the channel other than sensing the environment, and this sensing duration should be arranged accordingly to minimize false alarm probability. On the other hand, nodes need to transmit to deliver their collected information on the event. Hence, this constitutes a trade-off between sensing and event transport reliability. Moreover, real-time and delay-aware reliability requirements of some applications such as surveillance and target tracking may also introduce additional challenges on spectrum sensing. The effect of heterogeneous sensing duration on packet delivery ratio are also discussed in Section III-B.

\subsection{Spectrum Mobility with Primary User Arrival in CRSN}

When a licensed user arrival is detected, sensor nodes are required to stop transmitting data, vacate the channel and communicate over another free channel. To analyze the effects of licensed user arrival on throughput and packet delay, simulations are performed with different licensed user activity patterns. A licensed user is placed on each channel and transmits packets according to its activity pattern, so that its effect is clearly demonstrated. When the active period of licensed user is higher, finding an empty channel takes longer, i.e., $\tau_{S}^{h}$ increases. This delay has a direct effect on the network performance. Thus, to illustrate this, throughput and delay performances are given in Fig. 4 for varying $\tau_{L}^{o n}$ from 0 to $6 \mathrm{sec}$ with $\tau_{L}^{\text {off }}=6$ and $3 \mathrm{sec}$, respectively.

\subsubsection{Licensed User Activity Pattern}

It can be seen in Fig. 4(a) and 4(b), especially, with the increasing licensed user active period throughput drastically decays. Since increasing link delay due to spectrum handoffs, increases the RTT and do not allow SACK packets to reach nodes on time, the highest, i.e., $60 \%$, throughput degradation is experienced by CODA. On the other hand, ESRT and $(\mathrm{RT})^{2}$ inaccurately calculate their new reporting rates and fail to satisfy their reliability objectives. On the other hand, PSFQ experiences drop in the throughput above 50\%, in Fig. 4(b). Intrinsically, traditional WSN transport protocols do not consider spectrum mobility and spectrum sensing functionalities, and do not adapt to the licensed user activity and spectrum 
sensing periods to achieve reliability and congestion control. Furthermore, they regulate sending rate assuming all the nodes are exposed to the same channel condition and licensed user activity. However, in CRSN, it may not be always possible for all sensor nodes to have the same channel conditions, i.e., this may not be the case in heterogeneous CRSN [7].

While the licensed user active period increases and probability of finding a vacant channel decreases, i.e., $\tau_{S}^{h}$ increases, TP-CRAHN performs slightly better than WSN transport protocols due to its DSA-aware functionalities. It assumes presence of a predefined common control channel (CCC) for coordination of channel information. This approach cannot handle cases, where a large number of nodes attempt to transmit in a short amount of time. Since a single channel is used, it gets congested very quickly. In fact, this is highly likely in CRSN due to its bursty traffic nature and dense deployment. Furthermore, this approach causes high end-to-end latency due to contention delay. Since CRSN generally operates in a multi-hop manner, this delay may exceed tolerable delay bound for some time-critical applications. Moreover, most of the time, it may not be possible to find such a CCC available throughout the entire network.

The results in Fig. 4(c) and 4(d) indicate that the lack of ability to coordinate with DSA functionalities causes existing transport layer protocols for WSN to amplify end-toend delay. Because of extra time spent while retrieving the lost packets and its transmission rate regulation, observed delay for PSFQ is generally higher than others in Fig. 4(c). CODA experiences sharp increase in packet delay with the increase of licensed user active period $\left(\tau_{L}^{o n}\right)$ from 2 to $4 \mathrm{sec}$ in Fig. 4(d), since delays caused by DSA functionalities are attributed to congestion and evoke multiplicative decrease congestion avoidance, i.e., reducing rate to one half. Due to its real-time support, $(\mathrm{RT})^{2}$ performs relatively better than other protocols. Its performance can be improved by including spectrum sensing and mobility functionalities in the rate control algorithm.

\subsubsection{Number of Available Channels (Opportunistic Reli- ability)}

In Fig. 5, simulations are performed for varying number of available channels from 0 to 20 , with $\tau_{L}^{o n}=2 \mathrm{sec}$ and $\tau_{L}^{o f f}=3$ sec. RTT is highly variable in CRSN because spectrum handoff period vary with the number of available channels. Therefore, protocol-specific communication requirements cannot be met. In Fig. 5, CODA has the sharpest throughput increase with the number of available channels. As the number of available channels increases, initially estimated waiting period for SACK packets is approached. Furthermore, it can be observed for ESRT in Fig. 5 that, throughput improvement for increasing number of available channels from 4 to 6 is greater than the one achieved while increasing from 16 to 18 . This result reveals that the throughput gain saturates as the number of avail-

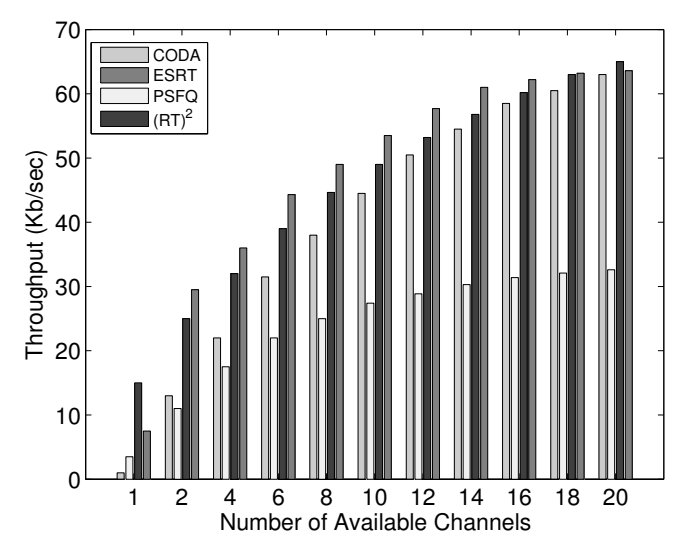

Figure 5: Throughput for varying number of available channels with $\tau_{L}^{o n}=2$ and $\tau_{L}^{o f f}=3 \mathrm{sec}$

able channels reaches to a certain level, which points out an important design trade-off to be further investigated.

In fact, higher number of available channels provides sensor nodes a flexible spectrum handoff operation, however, the impact of increasing available channels in throughput decreases as the throughput value without DSA is approached, i.e., gain obtained from increasing number of channel gets smaller. Therefore, access to sufficient number of channels, which is an important open issue to be determined, is essential for reliable and energy-efficient communication together with the efficient utilization of available spectrum in CRSN.

\subsubsection{Transient Effects of Licensed User Arrival}

Number of delivered packets at sink are examined to observe effects of varying licensed user activity with time. In these simulations, licensed user activity is increased gradually with time. Licensed user arrivals are scheduled at $15,45,55,75,80$ and $85 \mathrm{sec}$, while the number of available channels are $20,15,10,5,2,1$, respectively.

CODA shows the highest oscillatory behavior as seen in Fig. 6(a). Consecutive licensed user activities result in drastic decrease in the number of delivered packets by CODA, e.g., delivered packets reduces to one half after licensed user activities between 80 and 90 sec. At the end of consecutive licensed user arrivals, CODA decreases its rate due to non-arriving SACK packets, i.e., time-outs and attributing them as congestion. After licensed user activity is removed between 90 and $100 \mathrm{sec}$, CODA lacks a fast increase in the rate, hence, the number of delivered packets stays below $30 \%$ of its initial value. In $6(\mathrm{a})$, it can be seen that oscillatory behavior in the number of delivered packets is decreased with respect to CODA, however, it still changes between 350 and 550 packets/sec for ESRT, $(\mathrm{RT})^{2}$ and TP-CRAHN. After consecutive licensed user arrivals, ESRT and $(\mathrm{RT})^{2}$ show a sharp increase in the number of delivered packets, e.g., from 90 to $100 \mathrm{sec}$, goodput increases about $230 \%$ after three consecutive li- 


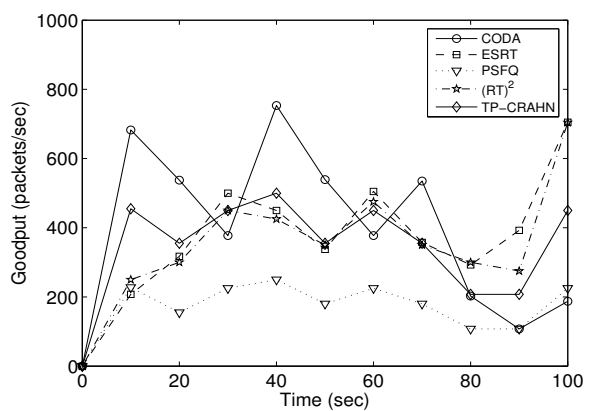

(a)

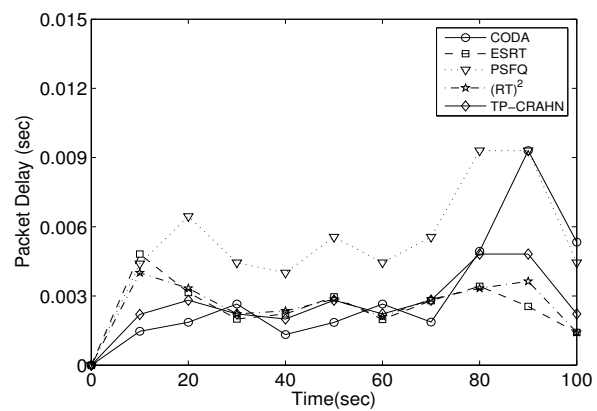

(b)

Figure 6: Variation of goodput (a) and delay (b) with respect to time for increasing licensed user activity.

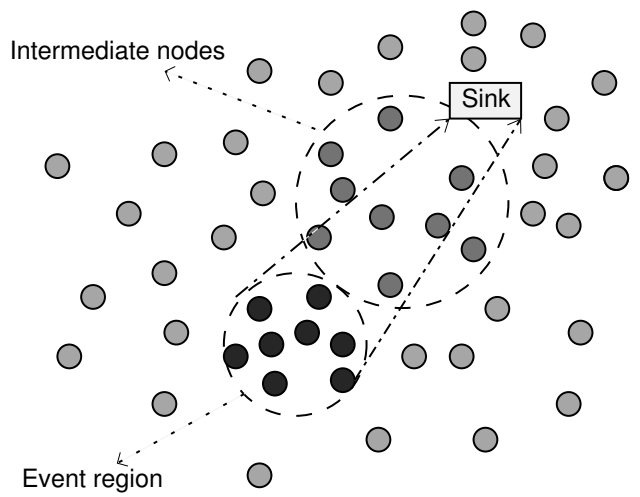

Figure 7: Event region and intermediate nodes in CRSN.

censed user arrivals in 70 and 90 sec. On the other hand, due to its adaptation to cognitive radio environment, TPCRAHN returns around its initial value (at $10 \mathrm{sec}$ ) at 100 sec.

In Fig. 6(b), average measured packet delay varies between 1 and $10 \mathrm{~ms}$ for each protocol. (RT) ${ }^{2}$ and ESRT outperform other protocols by their multiplicative rate increase property. CODA, which has an additive-increase multiplicative decrease (AIMD) rate control scheme, lacks fast recovery capability in case of connection disruptions due to spectrum handoffs. While licensed user activity increases in 70 and $90 \mathrm{sec}$, packet delay increases 5 times for CODA. Since PSFQ does not address for real-time event

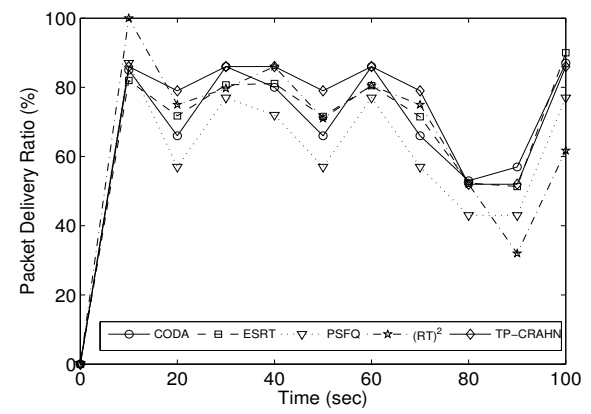

(a)

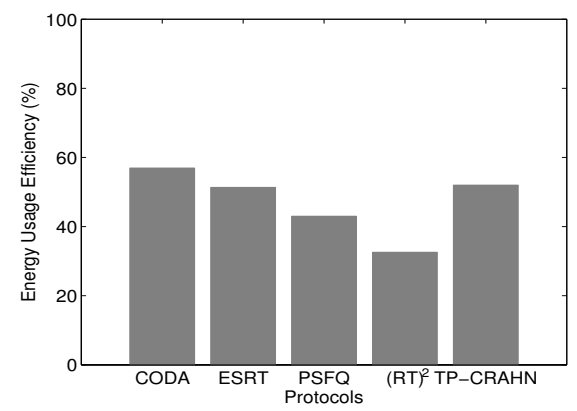

(b)

Figure 8: Transmitted packets to received packets ratio (a) and energy efficiency under high licensed user activity (b).

delivery, it is exposed to a greater delay than others. TPCRAHN, which also employs an AIMD rate control, relatively performs better than CODA and close to ESRT and $(\mathrm{RT})^{2}$.

\subsubsection{Spatially Varying Licensed User Arrival}

It is possible to have different licensed user activity characteristics at different regions of a cognitive radio network. Therefore, sensing duration required to detect licensed user activity may change. To illustrate this in CRSN, we obtain results of having different spectrum sensing durations at event region and by intermediate nodes as in Fig. 7.

In Fig. 8(a), it is shown that how packet delivery ratio changes in percentage, while different licensed user activities are observed in the network. Especially, between 80 and $90 \mathrm{sec}$ with increasing licensed user activity, packet delivery ratio reaches about half of the value measured without licensed user activity. Since TP-CRAHN is designed for cognitive radio ad hoc networks, nodes adjust their transmission periods as not to overlap with the spectrum sensing period of the next node on the path. Hence, it performs slightly better than traditional WSN transport protocols in general. On the other hand, PSFQ performs below the others with excessive packet drops.

In Fig. 8(b), it is shown that under heavy licensed user activity all of the protocols show an efficiency under $50 \%$. CODA, although exposed to degradation in packet delivery ratio up to $40 \%$ in Fig. $8(\mathrm{a})$, provides most efficient re- 
sults due to its sharp congestion avoidance mechanism. On the other hand, $(\mathrm{RT})^{2}$ aiming to provide real-time support, updates its reporting rate sharply with respect to previous decision interval's packet delay. As a result of this, if no licensed user activity is observed in current interval, it causes transmission of more than required packets and these packets cause congestion, hence, energy is wasted.

\subsection{Spectrum Coordination in $C R S N$}

Spectrum coordination is an important topic in cognitive radio networks especially in CRSN, since to be able to communicate with each other, sensor nodes should be operating on the same channel. Sensor networks need special interest on this issue due to limited resources and capabilities of sensor nodes, and reports of every node are important in event detection and tracking. To demonstrate the interaction of reliability and spectrum coordination, some parts of network left uncoordinated with the rest after spectrum handoff. These uncoordinated sensor nodes are selected in a random fashion, and their amount is given as percentage of the entire network.

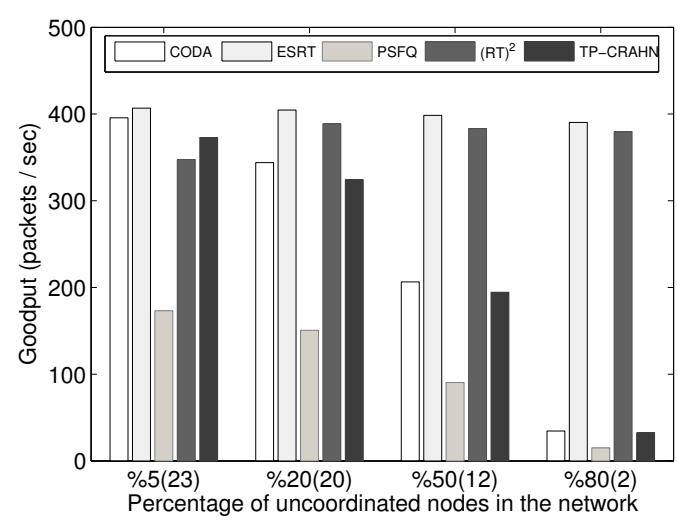

Figure 9: Variation of goodput with respect to percentage of network employing the same spectrum afterwards of spectrum handoff (in parenthesis number of coordinated event region nodes are given).

In Fig. 9, event-based protocols, i.e., ESRT and (RT) ${ }^{2}$ are shown to maintain goodput although unreachable nodes. However, since number of connected sources are decreased, to preserve reliability, reporting rates are re-adjusted to compensate for lost nodes. Therefore, coordinated nodes' resources are overused to satisfy reliability condition. This result shows that network lifetime is highly dependent on spectrum coordination between sensor nodes. On the other hand, for packet-based reliability considering protocols, goodput decreases below one quarter of its initial value (from 400 packets to about 50 packets per-second), while the percentage of uncoordinated nodes are increased. This is due to that fact that, independent from event detection and tracking requirements of sink, every node sends its own measurements and regulate its own transmission rate with respect to feedback packets coming from intermediate nodes. Therefore, when the available source node count decreases, reliability observed at sink decreases. Strong interaction between reliability, network resources and spectrum coordination can be inferred from the results obtained with the existing transport protocols as shown in Fig. 9.

\section{Shortcomings and Future Directions}

There is a delicate balance between reliability and energyefficiency, which has been the main focus of transport layer solutions proposed for sensor networks thus far [22]. While the same balance is also inherited by CRSN, dynamic spectrum management brings additional factors into this tradeoff. Hence, all of the challenges introduced in Section II and clarified with simulation results in Section III, along with the ones inherited from WSN and amplified by cognitive radio, must be addressed through the design of novel dynamic spectrum-aware CRSN transport protocols.

In this section, main results of the simulations are analyzed and elaborated, afterwards, open research directions for reliability and congestion control in CRSN are pointed out.

\subsection{Overview of Simulation Results}

Comprehensive simulations clearly point out the shortcomings of the currently proposed transport protocols, and show that WSN protocols suffer from cognitive cycle introduced with DSA.

First, CODA infers high licensed user activity as congestion, and regulates its transmission rate accordingly. Thus, CODA necessitates incorporation of DSA functionalities. Despite its relatively higher energy-efficiency, reliability stays far behind the requirements in CRSN. Additionally, CODA lacks a fast increase in the rate control afterwards of a high licensed user activity.

On the other hand, ESRT and (RT) $)^{2}$ provide applicationbased reliability. However, since inherent rate regulation simply does not take DSA challenges into account, extra delays and packet losses caused by cognitive cycle result in undesired behaviors such as transmission of redundant packets along with packet drops, i.e., depletion of confined resources. Although $(\mathrm{RT})^{2}$ considers real-time communication requirements, it encounters increase in the packet delay in the order of 5 , especially, while the number of available channels is extremely low and licensed user activity is high.

PSFQ aims to provide guaranteed packet delivery on the reverse path, i.e., from sink to sources. When available spectrum is bounded and high licensed user activity is exposed, it causes excessive losses while recovering missing packets from neighbor nodes. Especially, in heterogeneous licensed user activity case PSFQ underperforms.

TP-CRAHN provides adaptive sensing duration adjustment and congestion window scaling to handle DSA challenges. Nonetheless, it does not take inherent constraints and requirements of sensor networks into account, 
i.e., it does not consider energy-efficiency and many-to-one (source-to-sink) event delivery requirements. Additionally, end-to-end reliability notion does not suite very well in WSN, due to its bursty and many-to-one traffic nature.

Due to unawareness of cognitive cycle, and hence, misinterpretation of its functionalities, traditional WSN transport protocols do not function properly and achieve required energy-efficient reliable operation. New solutions are needed to overcome challenges introduced by cognitive radio. In simulation results, effects of generated extra delays and packet losses imposed by cognitive radio are explored. These results show the need for DSA-aware transport protocols for CRSN. TP-CRAHN is designed to operate on cognitive radio ad hoc networks and has features such as sensing duration minimization and adaptation to bandwidth variations. However, it does not consider inherent requirements of sensor networks and employs TCP congestion control algorithm, which is unsuitable for bursty many-to-one flow in CRSN.

\subsection{Open Research Issues}

In addition to energy-efficient and reliable communication requirements in WSN, emerging challenges of CRSN must be addressed through the design of novel, adaptive and spectrum-aware CRSN transport protocols. Clearly, none of the existing approaches devised for WSN can be directly employed in CRSN. Together with the extra delays and losses introduced by DSA functionalities in sensor nodes, reliability and congestion control in CRSN becomes an extremely challenging task. To this end, the open research issues for transport layer in CRSN are outlined as follows:

- Collaboration of transport layer protocols and DSA functionalities may provide efficient usage of available spectrum. Thus, cros-layer interaction of spectrum management and rate control mechanisms should be studied.

- There is a trade-off between required reporting rate and interference with licensed users. New metric definitions combining reliability and congestion with licensed user interference and spectrum characteristics are required.

- Varying spectrum availability and channel conditions require novel congestion avoidance algorithms. Higher licensed user activity may augment probability of congestion due to intermittent spectrum availability. On the other hand, congestion control mechanism should also take spectrum sensing duration into consideration together with varying licensed user activity, since sharp increases in number of packets injected into the network due to increasing transmission period may result in congestion. There may be bottlenecks, due to spatially varying spectrum sensing duration, i.e., licensed user activity, in the network. Therefore, congestion control mechanisms should consider sensing durations of the nodes on the event delivery path.

- For delay-bounded applications, real-time DSA-aware transport layer solutions should be developed. Based on acquired transmission behavior of licensed users, probabilistic rate control models may be developed. Probabilistic and predictive frameworks can be derived to reduce increasing delay with increasing licensed user activity. To this end, analytical models including licensed user activity with congestion and wireless loss probability could be developed.

- Spectrum coordination must be incorporated in energyefficient protocol stack in CRSN to provide reliable data delivery and congestion avoidance. Common control channel (CCC) is an alternative for spectrum coordination. However, since it requires high cost to lease a channel, it would amplify the cost of the sensor network [7]. Research on finding such a suitable CCC in unlicensed spectrum channels or coordination without CCC is required.

- Transport protocols should interact with sensing mechanism since it has a direct effect on throughput as it can be seen in Fig. 3 and utilize it to minimize licensed user interference and false detection probability while maximizing reliability. Hence, a cross-layer spectrum sensing and congestion control mechanism must be designed.

- Congestion control algorithms should also be adaptive to dynamically changing licensed user activity. Moreover, varying network capacity should be taken into consideration, since transmission more packets than network can tolerate will result in inefficient usage of limited resources of sensor nodes. Development of new congestion avoidance mechanisms incorporating licensed user activity and network capacity are essential. In addition, in order to utilize opportunistic spectrum availabilities and satisfy reliability requirements, DSA-aware fast rate increase algorithms are also required.

\section{Conclusion}

In this paper, CRSN challenges for reliable data delivery are explored, and it is shown by simulation experiments that the existing transport layer protocols for WSN and cognitive radio ad hoc networks are not suitable for CRSN. Furthermore, open research issues for CRSN transport layer are stated. Clearly, there is a need for energyefficient novel rate control, error recovery and congestion avoidance mechanisms which take challenges of CRSN into account. New CRSN transport protocols must consider communication impairments due to spectrum sensing and 
mobility such as excessive delays and packet losses incurred by cognitive cycle. Due to strict coupling of DSA challenges and transport layer, cross-layer paradigm seems promising in order to address CRSN challenges.

\section{Acknowledgment}

This work was supported in part by the Turkish Scientific and Technical Research Council under grant \#110E249 and by the Turkish National Academy of Sciences Distinguished Young Scientist Award Program (TUBA-GEBIP).

\section{References}

[1] S. Haykin, Cognitive Radio: Brain-empowered Wireless Communications, IEEE Journal on Selected Areas in Communications (JSAC), vol. 23, no. 2, pp. 201-220, Feb. 2005.

[2] I. F. Akyildiz, W. Y. Lee, M. C. Vuran, and S. Mohanty, NeXt Generation/Dynamic Spectrum Access/Cognitive Radio Wireless Networks: A Survey, Computer Networks Journal (Elsevier), vol. 50, no. 13, pp. 2127-2159, Sept. 2006.

[3] Q. Zhao, B. Sadler, A Survey of Dynamic Spectrum Access, IEEE Signal Processing Magazine, vol. 24, no. 3, May 2007.

[4] I. F. Akyildiz, W. Y. Lee, and K. Chowdhury, CRAHNs: Cognitive Radio Ad Hoc Networks, Ad Hoc Networks Journal (Elsevier), vol. 7 , no. 5 , pp. 810-836, Jul. 2009.

[5] A. M. Wyglinski, M. Nekovee, and Y. T. Hou, Cognitive Radio Communications and Networks: Principles and Practice, Elsevier, Amsterdam, The Netherlands, 2010.

[6] I. F. Akyildiz, W. Y. Lee, and K. Chowdhury, Spectrum Management in Cognitive Radio Ad Hoc Networks, IEEE Network, July-August 2009.

[7] O. B. Akan, O. B. Karli, and O. Ergul, Cognitive Radio Sensor Networks, IEEE Network, vol. 23, no. 4, pp. 34-40, July-August 2009.

[8] A. M. R. Slingerland, P. Pawelczak, R. V. Prasad, A. Lo, and R. Hekmat, Performance of Transport Control Protocol Over Dynamic Spectrum Access Links, in: Proc. IEEE DySPAN 2007, Dublin, Ireland, Apr. 2007.

[9] T. Issariyakul, L. S. Pillutla, V. Krishnamurthy, Tuning radio resource in an overlay cognitive radio network for TCP: Greed isn't good, Communications Magazine, IEEE , vol.47, no.7, pp.57-63, July 2009.

[10] Y. R. Kondareddy, and P. Agrawal, Effect of Dynamic Spectrum Access on Transport Control Protocol Performance, in: Proc. IEEE GLOBECOM 2009, Hawaii, USA, Dec. 2009.

[11] M. Di Felice, K. R. Chowdhury, W. Kim, A. Kassler, and L. Bononi, End-to-end protocols for Cognitive Radio Ad Hoc Networks: An evaluation study, to appear in Performance Evaluation Journal (Elsevier), 2010.

[12] W. Y. Lee and I. F. Akyildiz, Optimal Spectrum Sensing Framework for Cognitive Radio Networks, IEEE Trans. on Wireless Comm., vol. 7, no. 10, pp. 3845-3857, Oct. 2008.

[13] I. F. Akyildiz, B. F. Lo, and R. Balakrishnan, Cooperative Spectrum Sensing in Cognitive Radio Networks: A Survey, to appear in Physical Communication (Elsevier) Journal, 2011.

[14] C. Wan, S. B. Eisenman, and A.T. Campbell, CODA: Congestion Detection and Avoidance in Sensor Networks, in: Proc. ACM Sensys 2003, Los Angeles, USA, Nov. 2003.

[15] O. B. Akan and I. F. Akyildiz, "Event-to-Sink Reliable Transport in Wireless Sensor Networks, IEEE/ACM Trans. Networking, vol. 13, pp. 1003-1016, Oct. 2005.

[16] V. C. Gungor, O. B. Akan, and I. F. Akyildiz, A Real-Time and Reliable Transport Protocol for Wireless Sensor and Actor Networks, IEEE/ACM Trans. Networking, vol. 16, no. 2, pp. 359-370, Apr. 2008.
[17] C. Y. Wan, A. T. Campbell, and L. Krishnamurthy, PSFQ: A Reliable Transport Protocol for Wireless Sensor Networks, in: Proc. ACM WSNA 2002, Atlanta, USA, Sept. 2002.

[18] F. Stan and J. Heidemann, RMST: Reliable Data Transport in Sensor Networks, in: Proc. IEEE SNPA 2003, pp. 102-112, May 2003.

[19] K. R. Chowdhury, M. Di Felice, and I. F. Akyildiz, TP-CRAHN: A Transport Protocol for Cognitive Radio Ad-Hoc Networks, in: Proc. IEEE INFOCOM 2009, Apr. 2009.

[20] C. Luo, F. R. Yu, H. Ji, and V. C. M. Leung, Cross-Layer Design for TCP Performance Improvement in Cognitive Radio Networks, IEEE Trans. Vehicular Technology, vol. 59, no. 5, pp. 2485-2495, Jun. 2010.

[21] D. Sarkar, and H. Narayan Transport Layer Protocols for Cognitive Networks, in: Proc. INFOCOM 2010, USA, Mar. 2010.

[22] C. Wang, M. Daneshmand, B. Li, and K. Sohraby, A survey of Transport Protocols for Wireless Sensor Networks, IEEE Network, pp. 34-40, Vol. 20, No. 3, 2006.

[23] K. R. Chowdhury, I. F. Akyildiz, Cognitive Wireless Mesh Networks with Dynamic Spectrum Access, IEEE Journal on Selected Areas in Communications (JSAC), vol. 26, no. 1, pp. 168-181, Jan. 2008.

[24] Y. Shi, Y. T. Hou, and H. Zhou, Per-node-based optimal power control for multihop cognitive radio networks, IEEE Trans. Wireless Commun., vol. 8, no. 10, pp. 52905299, Oct. 2009.

[25] Y. Shi, Y. T. Hou, H. Zhou, and S. F. Midkiff, Distributed Cross-Layer Optimization for Cognitive Radio Networks, IEEE Trans. on Vehicular Technology, vol. 59, no. 8, pp. 4058-4069, Oct. 2010.

[26] D. Hu, S. Mao, Y. T. Hou, and J. H. Reed, Scalable Video Multicast in Cognitive Radio Networks, IEEE Journal on Selected Areas in Communications (JSAC), vol. 28, no. 3, pp. 434-444, Apr. 2010.

[27] Network Simulator - (ns-2), http://www.isi.edu/nsnam/ns/.

[28] D. Johnson, D. Maltz, Y. Hu, and J. Jetcheva, The Dynamic Source Routing Protocol for Mobile Ad Hoc Networks (DSR), IETF Internet draft, Feb. 2002. 